\title{
ARTICLE
}

\section{Using clozapine in clinical practice ${ }^{\dagger}$}

\author{
Ann M. Mortimer
}

\begin{abstract}
Ann M. Mortimer trained in
Leicester and Yorkshire prior to her first consultant post in West Yorkshire. She became a senior lecturer at Imperial College London in 1991 and took up her foundation chair at the Postgraduate Medical Institute, University of Hull, in 1996. Her research interests focus on psychosis, particularly schizophrenia and clinical psychopharmacology. She runs rehabilitation and assertive outreach services in Hull and East Yorkshire.

Correspondence Professor Ann M. Mortimer, Hertford Building, University of Hull, Cottingham Road, Hull HU6 7RX, UK. Email: a.m.mortimer@hull.ac.uk
\end{abstract}

${ }^{\dagger}$ See also pp. 250-255, this issue.

\begin{abstract}
SUMMARY
Clozapine remains underutilised as the only antipsychotic for treatment-resistant schizophrenia, despite liberal National Institute for Health and Clinical Excellence guidelines for its consideration. Bearing in mind its monitoring requirements and poor tolerability, suggestions are made for patients who fail to improve on other antipsychotic drugs. Clozapine may be offered to apparently unsuitable patients, although this is fraught with difficulty. A realistic appraisal of the alternatives is essential in this situation. Optimising plasma clozapine levels, alongside the use of rehabilitative interventions and adjuncts as necessary, will maximise efficacy, and there are numerous options to minimise sideeffects. Clozapine requires a lengthier trial than other antipsychotics and discontinuation should be avoided if possible, as the results are generally very poor. Established successful clozapine treatment can induce substantial functional gains which accrue with time: patients are retained in treatment and relapse is prevented. Such optimal outcomes justify the initial effort expended by all.
\end{abstract}

\section{DECLARATION OF INTEREST}

None.

Clozapine is the only antipsychotic drug licensed for treatment-resistant schizophrenia. Unfortunately, it has a number of problematic side-effects, principally neutropenia, which necessitates longterm monitoring.

Given the high prevalence of treatment resistance in schizophrenia (about one in three patients develops treatment resistance eventually) and the low numbers of patients being treated for it in the UK (about a third), the National Institute for Health and Clinical Excellence (NICE) issued guidelines that clozapine should be considered for patients who had failed to respond adequately to two sequential antipsychotic trials, one using an atypical, each given at therapeutic doses for 6-8 weeks (Barnett 2002). The key word is 'consider': the guidance does not mean that every patient should begin clozapine treatment if they fail to respond to two antipsychotic drugs given as described here. Indeed, this would trigger clozapine treatment much sooner and in far less ill patients than those included in seminal efficacy studies (Mortimer 2007). The original studies which demonstrated such markedly superior efficacy for clozapine included patients who met exceptionally narrow retrospective, cross-sectional and prospective criteria for treatment resistance (Kane 1988). There is no evidence that NICE-defined treatment resistance approximates to anything like this degree of severity. Indeed, although clozapine increases treatment retention and prevents relapse better than first-line treatment in patients without treatment resistance, it has no superior effects on psychopathology and induces worse metabolic side-effects (Metzer 2010). Given the difficulties encompassed by clozapine treatment, it is not surprising that clozapine continues to be underused (Nielsen 2010). In the USA, clozapine accounted for $11 \%$ of antipsychotic prescriptions in 1999: this fell to less than 5\% in 2006 (Kelly 2010).

The details of haematological monitoring and initial titration are well known and will not be reiterated here. This article will focus on how to choose patients for clozapine treatment and how to maximise its effects and minimise its drawbacks. Unfortunately, the cumbersome and anxiety-provoking nature of establishing clozapine treatment in the most difficult patients, who have the greatest need for it, deprives many of potentially substantial global clinical improvement.

I have 20 years' experience in the use of clozapine and have treated very large numbers of patients: what is offered here is a blend of research evidence and, hopefully, clinical acumen.

\section{Choosing suitable patients}

Consultants and their teams should go beyond NICE guidance, carefully appraising the patient's treatment history in order to identify reasons why the individual is failing to improve. The usefulness of defining in what way the patient is not getting better with standard treatment cannot be overemphasised, as it has significant ramifications for aetiology, treatment and prognosis. It is just as important that any strategy for partial response, including clozapine, comes with a predetermined 'exit strategy', and that the treating team is aware that the patient may experience a significant placebo effect which proves transient. Even so, it is important to bear in mind that official guidelines are heuristic and apply to groups of patients, not the individual patient. Guidelines cannot hope to reflect the complexity of cases seen in clinical practice: the skill and expertise of the consultant 
TABLE 1 Non-clozapine options for NICE treatment-resistant schizophrenia

\begin{tabular}{|ll|}
\hline Problem & Solution \\
\hline Partial/non-adherence & Depot antipsychotic \\
\hline Substance misuse/dependence & Treat this, recommend depot antipsychotic if there is non-adherence \\
\hline Extrapyramidal side-effects & Drop dose or try quetiapine, aripiprazole or olanzapine \\
\hline Positive symptoms & $\begin{array}{l}\text { Add another antipsychotic with a different mechanism of action } \\
\text { Try an anticonvulsant or lithium } \\
\text { Try an adjunct: mirtazapine, famotidine, minocycline, ondansetron, allopurinol, vitamin C, } \\
\text { hormone replacement therapy (suitable female patients only) }\end{array}$ \\
\hline Negative symptoms & $\begin{array}{l}\text { Primary symptoms: try low-dose amisulpride, SSRI, rehabilitation } \\
\text { Secondary symptoms: treat underlying causes }\end{array}$ \\
\hline Depression & Characterise and treat accordingly \\
\hline $\begin{array}{l}\text { Cognitive impairment, poor } \\
\text { personal and social function }\end{array}$ & Rehabilitation and social support \\
\hline Patient remains unsatisfactory & Clozapine \\
\hline
\end{tabular}

NICE, National Institute for Health and Clinical Excellence; SSRI, selective serotonin reuptake inhibitor.

and their team afford a broader and more flexible approach to issues of partial response.

\section{Poor adherence}

Non-adherence with oral medication is an obvious issue (Table 1). A trial of a depot antipsychotic should be completed in patients who have a history of medication non-adherence. Without the patient's agreement, this may not be possible unless the individual is detained for compulsory treatment. If there really is partial adherence or non-adherence, this scenario is likely to present itself sooner or later. Maintenance can be achieved by enacting a community treatment order (CTO), with use of the recall provision if the depot is refused.

\section{Substance misuse and dependence}

Although patients treated with clozapine often control their substance misuse or dependence better than those on standard treatment, the prime indication for clozapine remains treatmentresistant schizophrenia. Any antipsychotic drug will to some degree antagonise the psychotogenic properties of common illicit drugs such as stimulants and cannabis. The reliability of depot formulations may be particularly useful in this regard, again under CTO provisions if necessary. Whether the diagnosis is persistent substanceinduced psychotic disorder or illicit drugs are exacerbating idiopathic schizophrenia, the nature and degree of these disorders does fall within Mental Health Act provisions.

\section{Treatment intolerance}

Intolerance of conventional antipsychotics, in particular their extrapyramidal side-effects (EPSE), used to be a fairly common reason for prescribing clozapine. Clozapine is no longer unique in its freedom from EPSE: some atypicals, such as quetiapine, olanzapine and aripiprazole, have a very low propensity to cause EPSE. Nevertheless, clozapine is particularly indicated for patients with tardive dyskinesia or dystonia, although quetiapine is easier to use for both patient and doctor.

\section{Persistent positive symptoms}

In practice, persistence of positive symptoms is a very frequent reason for considering clozapine. A number of 'add on' options to current antipsychotic treatment may prove effective alternatives to clozapine for individual patients, although solid evidence for their effectiveness in groups of patients may be absent or limited. For example, despite the general disapproval of antipsychotic polypharmacy, it has been argued that augmentation of olanzapine with another antipsychotic is generally advantageous (Zink 2005). Adding lithium or anticonvulsant drugs has been studied to the point of systematic review and meta-analysis (Mortimer 2011), with generally disappointing results in groups of patients, although there have been positive individual trials, particularly with sodium valproate, lamotrigine and topiramate. There is some very limited evidence of the efficacy of non-steroidal anti-inflammatory drugs, whose theoretical therapeutic mechanism suggests that their advantages will be limited to the early stages of schizophrenia (Mortimer 2010). There are a number of other potential adjuncts, supported by small trials, including some placebo-controlled randomised controlled trials, whose putative utility is underpinned by plausible neuroscience. These include mirtazapine, famotidine, minocycline, ondansetron, allopurinol, vitamin $\mathrm{C}$ and hormone replacement therapy (Mortimer 2010). 
The stage at which one should give up trying alternatives to clozapine for the control of positive symptoms is an individual clinical decision. There should be a balance between, on the one hand, resorting early to a poorly tolerated drug and, on the other, protracted diminishing returns while exhausting the list of adjuncts.

\section{Persistent negative symptoms}

Primary negative symptoms

Core or primary negative symptoms comprise a loss of responsiveness to both external and internal cues, therefore poverty of thought and emotional blunting are considered primary. Core negative symptoms may be treated with selective serotonin reuptake inhibitor (SSRI) antidepressants or low-dose amisulpride, alongside psychological interventions (Mortimer 2001). Psychological interventions seek to provide apposite stimulation and to engage the patient in active mental activity, facilitating emotional responses through social contact and interaction. These are fundamental strategies in rehabilitation.

\section{Secondary negative symptoms}

Many patients present with 'secondary' negative symptoms, which are behaviourally similar to the core symptoms, but with other causes, including oversedation, depression, Parkinsonism or preoccupation with positive symptoms (Kelly 2010). Cognitive impairment, schizoid personality traits and institutionalisation are also likely to be relevant. Both primary and secondary symptoms tend to be closely associated with deterioration in personal and social function.

Oversedation is easily dealt with by dose reduction, switching to a less sedating antipsychotic such as aripiprazole, or cessation of coprescribed sedating medications.

Depression in schizophrenia requires essentially the same treatment strategies as depression without schizophrenia, although it may make sense to switch from a high-potency conventional antipsychotic to a low-potency atypical drug such as quetiapine, which is now licensed for bipolar depression.

Parkinsonian side-effects can be managed by lowering the dose or by switching to a bettertolerated antipsychotic such as quetiapine, olanzapine or aripiprazole. Long-term coprescription of anticholinergic drugs is not recommended, given their own side-effects and antagonism of antipsychotic therapeutic effects.

Preoccupation with unresolved positive symptoms may respond to the strategies mentioned above for persistent positive symptoms.
Cognitive impairment in schizophrenia is unfortunately not really amenable to any drug treatment, although better control of positive and negative symptoms is likely to bring about some indirect effects. There is no evidence that antidementia drugs are effective, and some, such as the $N$-methyl- $D$-aspartic acid antagonist mementine, could theoretically make positive symptoms worse. Cognitive function can be evaluated by the clinical psychologist, using formal premorbid and current IQ estimates: this will give a useful measure of cognitive decline and a guide to the personal and social function that can be expected. Appropriate rehabilitative strategies of engagement and social reinforcement may in some patients be usefully informed by clinicians with expertise in intellectual disability.

Many patients will require long-term assistance with independent living, the level of which should neither induce disability nor leave significant needs unmet. Similarly, patients with marked premorbid schizoid personality traits are likely to require long-term input. On the other hand, patients whose negative symptoms are secondary to institutionalised behaviour may demonstrate slow improvement which is maintained when interventions are reduced.

It is important that the treating team maintains realistic expectations and respects patient choice about what is provided. Whether the patient's mental health is sufficiently compromised to justify a trial of clozapine is a major clinical decision, which again involves balancing the risks and likely benefits.

\section{Barriers to clozapine treatment}

\section{Adherence}

Refraining from instituting clozapine because of an expectation that the patient will not adhere to treatment once back in the community is not an excuse to make no attempt to try it, particularly if the patient is currently detained for treatment under the Mental Health Act. Behavioural means such as the withdrawal of positive reinforcement (leave periods or access to cigarettes) are available, although treating teams may be reluctant to enforce them. Discussions regarding patient interests in the alternatives available are always useful, and the assistance of a competent clinical psychologist may be very helpful. It is important to set up reinforcement on at least a daily basis, and to allow the patient to change their mind about initial refusal of tablet consumption or venepuncture. There has to be some flexibility, for example allowing the patient today's leave if they now agree to swallow the clozapine tablet 
which they refused the previous night. Adherence to clozapine treatment may also be enhanced by using strategies derived from social psychology such as scarcity, consistency, authority and universality (Cialdini 2008). For example, it is worth emphasising to the patient that clozapine is a very special and costly treatment, which cannot be prescribed to all.

There is some evidence that patients maintain adherence with clozapine better than with other antipsychotic treatments (Wheeler 2009; Kelly 2010). Furthermore, there is limited recent evidence that co-prescription of depot antipsychotic treatment provides a 'safety net' for patients who repeatedly fail to take their clozapine properly (Kim 2010). The inception of the CTO is another tool in the adherence armamentarium: it is perfectly legitimate to recall patients to hospital because of non-adherence with clozapine: a 48hour break necessitates retitration in any case. Fortunately, such retitration may proceed much more rapidly than initial treatment. Assertive outreach teams are well placed to deliver daily doses of clozapine and make sure that these are consumed. It is possible that the inconvenience of recall may exceed the drawbacks of adherence from the patient's perspective.

\section{Suicide and other risks}

Patients with a history of suicidality, hostility and aggression may benefit more from clozapine than from other treatments (Kelly 2010). Unfortunately, such patients may be deemed to be at particularly high risk of non-adherence to medication and therefore considered unsuitable for clozapine treatment. Clinicians may wish to minimise the risks by commencing clozapine within an inpatient admission.

\section{Neutropenia with clozapine or other medications}

Sometimes it is possible to treat patients with clozapine when there is much to discourage this. For example, patients who have developed neutropenia may be rechallenged, particularly if the onset of neutropenia was after 6 months of treatment, because further clozapine treatment does not appear to be associated with agranulocytosis (Kelly 2010). Furthermore, some ethnic groups, particularly of African derivation, have neutrophil counts consistently lower than their White counterparts, a condition known as benign ethnic neutropenia, without, apparently, any greater risk of infection overall. There is another haematological condition, benign fluctuating neutropenia, in which the neutrophil count regularly drops in and out of the 'red' range but with no impact on physical health. Lithium can be prescribed to patients with a history of neutropenia as a result of clozapine or other medication or idiopathic neutropenia. Lithium is associated with an increase in the leucocyte count, although there is no evidence that this will prevent neutropenia while on clozapine.

There is a very small series of case reports describing patients treated with granulocyte stimulating factor on a prophylactic basis alongside clozapine, with some success. Granulocyte stimulating factor may also be used as a 'rescue' strategy in benign fluctuating neutropenia, alongside a lower threshold for the discontinuation of clozapine.

Securing clozapine treatment in patients with neutropenia is not easy. The decision to proceed is best informed by a realistic consideration of the alternatives, such as the prospect of several decades of unremitting psychosis in secure care, with all its adverse implications for the patient's quality of life. A sympathetic and constructive local consultant haematologist is essential if the numerous hurdles to clozapine treatment are to be overcome. Even if the treating team, haematologist, relatives/carers and patient agree that the potential benefits of clozapine treatment outweigh the potential risks, treatment automatically becomes 'off licence'. Treatment must therefore be specially authorised by the UK Clozaril Patient Monitoring Service (CPMS) or equivalent. Subsequently, the treatment proposal must go through often lengthy and labour-intensive trust governance processes: if the patient is detained under the Mental Health Act, lacks capacity, or both, there are substantial additional considerations. Opinions must be sought, forms filled in and meetings convened. There will or may be involvement from relatives, informal and professional carers, pharmacists, drug and therapeutics committee representatives, National Health Service managers, advocates, second opinion doctors, solicitors, and individuals holding lasting power of attorney, many of whom may wish to attend 'best interests' meetings on behalf of the patient. A high level of commitment on the part of the treating team is crucial to implementation of clozapine treatment in these circumstances, and after the treatment begins the team must prove able to contain their own and everybody else's anxieties. These processes become easier with practice.

\section{Getting the best out of clozapine}

It is always a serious decision to start clozapine: the patient will have exhausted alternative options while remaining unacceptably compromised. There is, therefore, no point in not giving 
clozapine a fair trial. Unlike other antipsychotics, the benefits of clozapine appear to continue to accrue as months and years of treatment are completed (Wheeler 2009). Similarly, the burden of compromised personal and social function that characterises treatment-resistant schizophrenia will be significantly or substantially relieved by successful clozapine treatment eventually: patients may progress to less intensively supported accommodation where they can exercise more choice and enjoy more independence. The most likely explanation for this is that clozapine induces better control of positive and negative symptoms to the extent that the patient can gradually engage with their environment and the people in it to a far more productive degree. Some return of insight can be particularly influential in this regard. Therefore, once started, clozapine should be persisted with, if possible, for at least several months before a decision to stop for poor efficacy. There are a number of strategies to ensure that the trial is a fair one.

\section{Clozapine levels}

Clozapine is unique in that a 'therapeutic threshold' has been defined for it: this affords a great deal of clinical utility. To be interpretable, the level should represent a 'trough' level and should arrive with information about dose, date and time of last dose before the blood sample was taken, as well as the date and time of the sample itself. It is worth emphasising that there is no imperative to exceed the therapeutic threshold unless patients are failing to improve at levels below it. To give an improving patient a therapeutically excessive dose will merely lead to more side-effects and interactions, not to mention expense. Treatment should be determined by the patient's response, not the clozapine level.

Because many clozapine side-effects are partially dose responsive, a clozapine level in a patient who is improving but nonetheless cannot tolerate the drug can give an indication of how much room there is to manoeuvre in terms of dosage reduction. There is no evidence to suggest that clozapine is unlike other antipsychotic treatments, in the sense that there needs to be a higher initial treatment dosage and a sometimes much lower maintenance dosage (e.g. Yamin 2010).

It is important to remember that stopping smoking can have quite profound effects on raising the clozapine level. In my experience, a substantial cut in dosage is usually required - as much as half the dose in a heavy smoker of 30 cigarettes a day. Other interactions may also vary the clozapine level by inducing or inhibiting hepatic cytochrome $\mathrm{P}$ enzymes. Inhibitors include caffeine, some SSRIs, antibiotics and antifungals; inducers include carbamazepine (which obviously should not be given with clozapine anyway, as it is also associated with neutropenia), phenytoin, omeprazole, St John's Wort and barbecued meat. It makes sense to review changes to patients' medication in order to determine possible influences on the clozapine level.

Furthermore, clozapine may displace other protein-bound drugs, thus raising their serum level. This is important with drugs such as warfarin and digoxin, since increasing their availability too much may have very serious consequences. Moreover, if possible, co-prescription of drugs that share aspects of clozapine's multiple receptor affinities should be avoided. These include drugs with anticholinergic, antihistaminergic and anti-adrenergic activity. Such drugs are likely to enhance clozapine-induced anticholinergic sideeffects such as constipation, antihistaminergic side-effects such as sedation, and anti-adrenergic side-effects such as hypotension.

If a patient is failing to respond to clozapine, repeated demonstration of therapeutic levels above the threshold can provide justification for prescribing additional psychotropic medication or, alternatively, stopping clozapine treatment. The trough level will generally demonstrate a norclozapine level about half the clozapine level: if they are the same, the patient may not have taken their last dose. Despite some interesting properties, norclozapine by itself does not act as an antipsychotic.

A clozapine level of zero can help patients and psychiatrists address non-adherence in a very direct fashion. Indeed, it is useful to take blood for a clozapine level on the admission of patients experiencing a relapse. Official printed information is often perceived to be more valid than verbal information and is more difficult to argue with.

\section{When monotherapy is insufficient}

A failure of response to adequate clozapine levels is always disappointing (Table 2). Essentially, there is little difference between clozapine and any other antipsychotic drug in the types of therapeutic interventions available for partial response or nonresponse, as discussed earlier (Table 1). Again, it is important to specify in what way the treatment is failing and to address that.

\section{Positive symptoms}

Positive symptoms are particularly problematic, and there is no standard strategy with any strong evidence. A number of studies have suggested the utility of adding another antipsychotic drug to 


\begin{tabular}{|c|c|}
\hline Problem & Solution \\
\hline Clozapine level suboptimal & Adjust dose above 'therapeutic threshold', check interactions \\
\hline Positive symptoms with adequate clozapine level & Use options from Table 1 \\
\hline Negative symptoms & Use options from Table 1, check levels if using an SSRI \\
\hline Cognitive impairment, poor function & Rehabilitation, social support, wait and see \\
\hline Depression & Characterise and treat accordingly; if 'awakenings', close personal support, decelerate expectations \\
\hline Neutropenia & $\begin{array}{l}\text { Consider risks and benefits of initiation/rechallenge, seek consultant haematology support, consider } \\
\text { granulocyte stimulating factor, 'rescue' contingency or prophylaxis }\end{array}$ \\
\hline Eosinophilia/thrombocytopenia & Stop until resolved \\
\hline Fever & Antipyretic, monitor for signs of infection, check WCC, ECG, creatine phosphokinase \\
\hline Tachycardia & ECG, monitor for cardiac symptoms, try a beta blocker \\
\hline Myoclonic jerks & Drop dose if possible, small dose of clonazepam at night \\
\hline Clozapine dose above $600 \mathrm{mg}$ daily or level $>0.60$ & Sodium valproate seizure prophylaxis \\
\hline Oversedation & $\begin{array}{l}\text { Drop dose, split (small dose in the morning, larger at night) or give dose all at night, stop other sedating } \\
\text { medication }\end{array}$ \\
\hline Constipation & Dietary advice, laxatives, drop dose if possible \\
\hline Urinary incontinence & Assess, use anticholinergic smooth muscle relaxant if appropriate, drop dose if possible \\
\hline Sialorrhoea & Hyoscine, drop dose if possible \\
\hline Weight gain, hyperglycaemia, dyslipidaemia & Dietary advice, exercise, metformin, add aripiprazole \\
\hline Risk of unintentional pregnancy & Full discussion with patient, contraceptive advice or referral if appropriate \\
\hline
\end{tabular}

ECG, electrocardiogram; SSRI, selective serotonin reuptake inhibitor; WCC, white cell count.

clozapine: it has been concluded that this may be worth trying in the individual patient, but evidence of efficacy across groups of patients is generally poor (Cipriani 2009). Again, there should be an exit strategy: there can be significant but shortlived placebo effects, which should not be mistaken for genuine efficacy.

Regarding cognitive-behavioural therapy (CBT) for positive symptoms, despite NICE recommendations, there is a body of opinion that CBT is not, in fact, efficacious (McKenna 2010). Thus, it seems highly unlikely that CBT will have much impact on positive symptoms in patients who are also resistant to both ordinary antipsychotics and clozapine, although there is a single small trial which claims that CBT does work in such cases (Barretto 2009).

\section{Negative symptoms}

It is now considered that clozapine does not alleviate the so-called primary or core negative symptoms of schizophrenia, but reduces secondary negative symptoms. Selective serotonin reuptake inhibitors and low-dose amisulpride can both be added to clozapine. The SSRIs should be used with caution as they can exacerbate positive symptoms. Furthermore, fluvoxamine, fluoxetine, paroxetine and sertraline (in that order of magnitude) elevate clozapine levels. Citalopram does not have this effect, and there are as yet no data on escitalopram.
Thus, although SSRIs may offset sedation and possibly weight gain, they may exacerbate or induce other problematic clozapine side-effects.

Regarding secondary negative symptoms, clozapine can be highly effective for unresolving positive symptoms masquerading as the negative variety. Unlike conventional antipsychotic drugs, clozapine is not depressogenic and it does not cause EPSE. By contrast, clozapine tends to be very sedating. This can be minimised by reducing the dose if possible, giving the whole dose at bedtime or splitting the dose (the morning dose smaller than the evening dose), and avoiding co-prescription of other sedating drugs. The effects of clozapine on cognition, personal and social function are likely to be indirect, slowly accruing over time as discussed earlier.

Rehabilitative interventions are just as appropriate for patients treated with clozapine: the provision of structure to daily living, stimulation, encouragement of engagement and design of reinforcement may induce motivation, emotional responsiveness and non-pathological spontaneous mental activity. Again, this is a long-term process, which may require indefinite maintenance.

\section{Depression and 'awakenings'}

Sometimes patients fail to make a full response because of affective symptoms. Patients tending towards schizoaffective disorder may benefit from 
mood-stabilising drugs, particularly if their mood is elevated. 'Depression' covers a multitude of sins, encompassing everything from understandable misery, through adjustment disorder and major depression. As a general rule, there is no reason why treatment should not proceed as if the patient did not have schizophrenia, as discussed above, with the usual blend of pharmacological and psychological support.

An exception to this is the 'awakenings' reaction (Weiden 1996; Duckworth 1997). Insight induced by clozapine treatment can be very painful when it includes the realisation of loss of mental health over many years, while still having to live with the continuing presence of schizophrenia (Rudge 2001). Related to this is the understandable optimism of mental health professionals and relatives, which often precipitates expectations which the patient feels unable to fulfil. Not infrequently, patients decide to cease clozapine treatment and it sometimes proves impossible to get them back on it. In the end, the treating team must respect the patient's decision, while making sure that the door to clozapine is kept open. Other patients become very depressed, occasionally to the point of suicidality. In my experience, very skilled psychotherapeutic support is of much more importance than pharmacological strategies in these circumstances.

\section{Monitoring clozapine treatment and dealing with side-effects}

The realisation that antipsychotics treatment of any kind can have profound side-effects has led to something of an explosion in the monitoring considered necessary. Clozapine treatment is already intensively monitored for neutropenia: extensive requirements in addition to this are likely to act as yet another disincentive to its use. The CPMS recommends a physical examination and the consideration of an electrocardiogram (ECG) as well as a pre-treatment blood count (Novartis 2010).

There is an argument that excessive monitoring during treatment merely serves to raise problems which may not be treated effectively, weight gain being a prime example. Furthermore, the detection of isolated anomalies which usually prove clinically irrelevant may encourage the clinician to focus on treating them, while losing sight of the importance of the patient's mental health. Decisions must be made on the balance of risks and benefits: satisfactory mental health in a treatment-resistant patient should be accorded substantial weight. This is especially so given the often catastrophic effects of stopping clozapine treatment.

\section{Other haematological anomalies}

It is recommended that both eosinophilia $(>3.0 \times 109 / \mathrm{l})$ and thrombocytopenia $(<50 \times 109 / \mathrm{l})$ indicate clozapine discontinuation, although it can be restarted when these have resolved.

\section{Fever}

Patients during the first 3 weeks of treatment commonly experience a fever: this is likely to prove benign, but there should be awareness of rare causes such as myocarditis and neuroleptic malignant syndrome. An ECG and creatine phosphokinase level should be ordered. Common infections should be looked for, as there is an obvious risk of neutropenia, although most neutropenias present silently rather than as overt infection. Fever and tachycardia together would suggest an infection, although both are early clozapine side-effects. Antipyretic medication may be given, but most fevers resolve spontaneously.

\section{Tachycardia}

Cardiac symptoms of any kind should be taken seriously as there is a (fortunately rare) association of clozapine treatment with myocarditis and cardiomyopathy. Persistent tachycardia in the first 2 months of treatment is associated with this; even so, tachycardia is very common, with a quarter of patients affected overall. The CPMS recommendations imply that myocarditis should be suspected and clozapine stopped if tachycardia is accompanied by other cardiac symptoms or if such symptoms occur by themselves (Novartis 2010). Some services carry out routine evaluation of cardiac enzymes and echocardiograms in patients treated with clozapine, but I am not aware of any evidence for or against this practice. Isolated tachycardia may respond to beta-adrenergic antagonists such as atenolol, which will necessitate monitoring of blood pressure. Reduction in dose, assuming that a lower dose will remain effective, is a better solution if possible. The opinion of a cardiologist should be sought if necessary.

Clozapine, like other antipsychotic drugs, may be associated with depression of the S-T segment, flattening or inversion of T-waves, and QTc prolongation on the ECG. In the absence of cardiac symptomatology there is no evidence that such anomalies herald myocarditis, cardiomyopathy or life-threatening arrhythmias, but it makes sense to keep the patient's cardiac status under review and to repeat the ECG in due course.

\section{Epileptic seizure}

Clozapine reduces seizure threshold: the incidence of fits is about $5 \%$ at a level of $0.6 \mathrm{mg} / \mathrm{l}$. It is prudent 
to add an anticonvulsant (but not carbamazepine) if the clozapine level approaches this threshold, as the experience of an epileptic fit is hardly likely to encourage future adherence. Sodium valproate may act as an antipsychotic adjunct, but again, a lower dose and level of clozapine would be the best option. Some patients experience myoclonic jerks: if problematic and persistent, a small bed-time dose of clonazepam may help.

\section{Anticholinergic effects}

The anticholinergic side-effects of clozapine particularly affect the gastrointestinal tract, although urinary overflow incontinence and retention, prostatism and glaucoma may also present. Clozapine-induced constipation requires attention to diet, but may require long-term osmotic laxatives. Other drugs with anticholinergic effects should be avoided. Urinary incontinence may also result from oversedation or seizures, especially if nocturnal, or may be contributed to by constipation. Nocturnal incontinence or urinary frequency can be managed with smooth muscle relaxant anticholinergic drugs such as oxybutynin and flavoxate, but these can exacerbate constipation and overflow incontinence, so it is important to evaluate thoroughly the patient's side-effects and response to any treatments.

\section{Sialorrhoea}

Hypersalivation is a common clozapine side-effect and one that patients find particularly troublesome. It derives from muscarinic agonism (Henderson 2006), which in the salivary gland appears to overcome clozapine's anticholinergic effects. Numerous remedies, mostly anticholinergic and antimuscarinic, have been recommended but with little evidence (Taylor 2009). Hyoscine $300 \mathrm{mcg}$ three times daily is most commonly prescribed.

\section{Metabolic side-effects}

Metabolic side-effects such as weight gain, diabetes and dyslipidaemia are a problem with many antipsychotic drugs, but clozapine is particularly prone to inducing them. Many patients practise lifestyle choices which induce or increase the risk of weight gain, metabolic side-effects and related endocrinological and cardiovascular complications: consumption of 'junk food', lack of physical activity and smoking.

Despite numerous published guidelines, most metabolic side-effects remain undetected and untreated: morbid obesity and diabetes in patients must be seen in the context of escalating prevalence rates in the general population. Metformin, particularly when combined with diet and exercise, may be a clinically useful treatment (Carrizo 2009; Frighi 2010). The addition of aripiprazole may lower weight, cholesterol and triglycerides, although there seem to be no further benefit for the symptoms of schizophrenia (Henderson 2006). Seventy-five per cent of patients so treated may lose weight and allow the clozapine dose to be reduced by a fifth, possibly offsetting other side-effects as well (Karunakaran 2007).

\section{Sedation}

Sedation is a very common side-effect: indeed, clozapine will interact with any other drug which has cerebral depressant properties such as benzodiazepines and alcohol to produce even more sedation. Splitting the dose may address morning oversedation by giving the patient most of their clozapine at night. Some patients do better on a once-daily night-time dose. Reducing the dose as much as possible is the simplest alternative, as long as therapeutic efficacy is preserved.

\section{Fertility}

Hyperprolactinaemia, which causes low fertility, is a common side-effect of many antipsychotics, but it is not induced by clozapine. Consequently, women switched to clozapine from antipsychotics that induce hyperprolactinaemia should be told that unprotected intercourse may well result in pregnancy.

Ideally, a detailed discussion regarding all aspects of becoming a parent should be offered to women taking clozapine. Consequential matters will probably include the parental relationship, contraceptive precautions, parenting capacity and the heritability of schizophrenia.

Clozapine has no known deleterious effects on unborn babies at any stage of gestation but, not surprisingly, there are very few data from which to draw conclusions. It is established that clozapine passes into breast milk, possibly at concentrated levels, and therefore breastfeeding is not recommended.

\section{Clozapine discontinuation}

There is no evidence that clozapine is curative with respect to treatment resistance; in other words, that if clozapine is discontinued, the patient may be adequately treated with any other antipsychotic. Sudden discontinuation of clozapine is very often followed by rebound psychosis, which can be severe and very difficult to treat. Rebound psychosis can be complicated by cholinergic rebound, which can cause nausea, vomiting, diarrhoea, headache, sweating, restlessness and agitation. This at least can be treated easily with an anticholinergic drug. 


\section{MCO answers}

$\begin{array}{lllll}1 b & 2 a & 3 d & 4 c & 5 d\end{array}$
Even so, clozapine discontinuation should take place gradually, unless this cannot be avoided (for example, if clozapine has to be stopped abruptly because there is neutropenia).

Justification for stopping clozapine must rest, as must discontinuation of any drug, on lack of efficacy, tolerability, or both. Given the cumbersome and inconvenient nature of clozapine treatment for the patient and for the treating team, it is likely that a low threshold for stopping clozapine may mirror a high threshold for starting it. Indeed, there is a little evidence that clozapine may be discontinued for no apparent reason (Mortimer 1997). Therefore, a determined approach to overcoming poor efficacy and tolerability is required on the part of all concerned.

\section{Conclusions}

Clozapine remains the only effective drug for treatment-resistant schizophrenia. Its effectiveness can be maximised by serum level monitoring and the use of rehabilitation strategies and other psychotropic drugs if necessary. Its tolerability is far from ideal, but there are ways to manage its side-effects, including neutropenia. Clozapine is considered to be underutilised, although the NICE definition of treatment resistance deviates markedly from that employed in seminal efficacy studies.

Establishing and maintaining suitable patients on clozapine treatment is not easy and requires a high level of commitment, persistence and tenacity on the part of all concerned. Attempts to do this without sufficient knowledge and expertise are unlikely to succeed. Nevertheless, the mental health and functional gains made by patients treated successfully can be dramatic and continue to accrue over many years. This makes clozapine treatment well worth the considerable efforts involved.

\section{Acknowledgements}

Thanks to the anonymous peer reviewers for their assistance.

\section{References}

Barnett D (2002) Guidance on the Use of Newer (Atypical) Antipsychotic Drugs for the Treatment of Schizophrenia. National Institute for Clinical Excellence.

Barretto EM, Kayo M, Avrichir BS, et al (2009) A preliminary controlled trial of cognitive behavioural therapy in clozapine resistant schizophrenia. Journal of Nervous and Mental Disease 197: 865-8.

Carrizo E, Fernandez V, Connell L, et al (2009) Extended release metformin for metabolic control assistance during prolonged clozapine administration: a 14-week, double-blind, parallel group, placebocontrolled study. Schizophrenia Research 113: 19-26.

Cialdini R (2008) Influence: Science and Practice (5th edn). Prentice Hall.

Cipriani A, Boso M, Barbui C (2009) Clozapine combined with different antipsychotic drugs for treatment-resistant schizophrenia. Cochrane Database of Systemic Reviews 3: CD006324.
Duckworth K (1997) Lost time, found hope and sorrow: the search for self, connection, and purpose during 'awakenings' on the new antipsychotics. Harvard Review of Psychiatry 5: 227-33.

Frighi V (2010) Medical side-effects of antipsychotic agents: hyperprolactinaemia and metabolic disorders. In Therapeutic Strategies in Schizophrenia (eds AM Mortimer, PJ McKenna): 1-20. Clinical Publishing.

Henderson D, Kunkel L, Nguyen D, et al (2006) An exploratory openlabel trial of aripiprazole as an adjuvant to clozapine therapy in chronic schizophrenia. Acta Psychiatrica Scandinavica 113: 142-7.

Kane J, Honigfeld G, Singer J, et al (1988) Clozapine for the treatmentresistant schizophrenia. A double-blind comparison with chlorpromazine. Archives of General Psychiatry 45: 789-96.

Karunakaran K, Tungaraza T, Harborne G (2007) Is clozapine-aripiprazole combination a useful regime in the management of treatment-resistant schizophrenia? Journal of Psychopharmacology 21: 453-6.

Kelly DL, Buchanan RW (2010) The current status of clozapine in and beyond treatment-resistant schizophrenia. In Therapeutic Strategies in Schizophrenia (eds AM Mortimer, PJ McKenna): 157-72. Clinical Publishing.

Kim SH, Jung D, Ahn YM, et al (2010) The combined use of risperidone long-acting injection and clozapine in patients with schizophrenia nonadherent to clozapine: a case series. Journal of Psychopharmacology 24: 981-6.

McKenna PJ (2010) Cognitive therapy for schizophrenia: does it work? In Therapeutic Strategies in Schizophrenia (eds AM Mortimer, PJ McKenna): 75-86. Clinical Publishing.

Metzer HY, Bobo WV, Lee MA, et al (2010) A randomised trial comparing clozapine and typical neuroleptic drugs in non-treatment resistant schizophrenia. Psychiatry Research 177: 286-93.

Mortimer AM (1997) A clozapine cohort: outcome and issues of concern. Human Psychopharmacology 12: 361-3.

Mortimer AM (2007) Optimising drug treatments in rehabilitation practice. Progress in Neurology and Psychiatry 11: 6-10.

Mortimer AM (2010) When monotherapy is insufficient. In Therapeutic Strategies in Schizophrenia (eds AM Mortimer, PJ McKenna): 139-56. Clinical Publishing.

Mortimer AM (2011) The magic shotgun: does it fit the clinician, and will it point at schizophrenia? In Schizophrenia Spectrum Disorders: Advances and New Directions (vol. 3) (ed M Ritsner). Springer.

Mortimer AM, Spence S (2001) Managing Negative Symptoms of Schizophrenia. Science Press.

Nielsen J, Dahm M, Lublin H, et al (2010) Psychiatrists' attitude towards and knowledge of clozapine treatment. Journal of Psychopharmacology 24: 965-71.

Novartis (2010) Clozaril $25 \mathrm{mg}$ and $100 \mathrm{mg}$ tablets. The electronic Medicines Compendium (http://www.medicines.org.uk/emc/document. aspx?documentid=1277)

Rudge T, Morse K (2001) Re-awakenings? A discourse analysis of the recovery from schizophrenia after medication change. Australian and New Zealand Journal of Mental Health Nursing 10: 66-76.

Taylor D, Paton C, Kapur S (2009) The Maudsley Prescribing Guidelines (10th edn). Informa Healthcare.

Weiden P, Aquila R, Standard J (1996) Atypical antipsychotic drugs and long-term outcome in schizophrenia. Journal of Clinical Psychiatry 57 (suppl 11): 53-60.

Wheeler A, Humberstone V, Robinson G (2009) Outcomes for schizophrenia patients with clozapine treatment: how good does it get? Journal of Psychopharmacology 23: 957-65.

Yamin S, Vaddadi K (2010) Are we using excessive neuroleptics? An argument for systematic dose reduction in stable patients with schizophrenia with specific reference to clozapine. International Review of Psychiatry 22: 138-47

Zink M (2005) Augmentation of olanzapine in treatment resistant schizophrenia. Journal of Psychiatry and Neuroscience 30: 409-15. 
MCQs

Select the single best option for each question stem

1 Clozapine is not associated with:

a eosinophilia

b hypothyroidism

c myocarditis

d tachycardia

e type II diabetes.

2 Clozapine should not be given if the patient:

a develops tachycardia, dyspnoea and chest pain

b has a BMl of $35 \mathrm{~kg} / \mathrm{m}^{3}$

c has an eosinophil count of $2.9 \times 109 / 1$

$\mathrm{d}$ has benign fluctuating neutropenia

e lacks parenting capacity.
3 For persistent positive symptoms, the following are of no merit:

a a trial of anticonvulsant medication

b a trial of depot medication

c a trial of two antipsychotic drugs with differing mechanisms of action

d low-dose amisulpride monotherapy

e urinary screens for illicit drugs.

4 Patients on clozapine treatment experience the following:

a benefit from 'awakenings' experiences

b direct benefit on primary negative symptoms

c gradual global functional improvement over a number of years

d reduced risk of major depressive episode

e remediation of premorbid cognitive impairment.
5 You wish to rechallenge a patient who developed neutropenia on clozapine after 5 years. It is always essential to involve the following people except:

a hospital pharmacy staff

b the care coordinator

c the clozapine monitoring agency

$\mathrm{d}$ the independent mental health capacity advocate

e the patient.

\section{'Why I wrote The Yellow Wallpaper?', by Charlotte Perkins Gilman}

\section{Selected by Femi Oyebode}

Many and many a reader has asked that. When the story first came out, in the New England Magazine about 1891, a Boston physician made protest in The Transcript. Such a story ought not to be written, he said; it was enough to drive anyone mad to read it.

Another physician, in Kansas I think, wrote to say that it was the best description of incipient insanity he had ever seen, and - begging my pardon - had I been there?

Now the story of the story is this:

For many years, I suffered from a severe and continuous nervous breakdown tending to melancholia - and beyond. During about the third year of this trouble I went, in devout faith and some faint stir of hope, to a noted specialist in nervous diseases, the best known in the country. This wise man put me to bed and applied the rest cure, to which a still good physique responded so promptly that he concluded there was nothing much the matter with me, and sent me home with solemn advice to "live as domestic a life as far as possible," to "have but two hours' intellectual life a day," and "never to touch pen, brush or pencil again as long as I lived." This was in 1887.

I went home and obeyed those directions for some three months, and came so near to the border line of utter mental ruin that I could see over.
Then, using the remnants of intelligence that remained, and helped by a wise friend, I cast the noted specialist's advice to the winds and went to work again - work, the normal life of every human being; work, in which is joy and growth and service, without which one is a pauper and a parasite; ultimately recovering some measure of power.

Being naturally moved to rejoicing by this narrow escape, I wrote The Yellow Wallpaper, with its embellishments and additions to carry out the ideal (I never had hallucinations or objections to my mural decorations) and sent a copy to the physician who so nearly drove me mad. He never acknowledged it.

The little book is valued by alienists and as a good specimen of one kind of literature. It has to my knowledge saved one woman from a similar fate - so terrifying her family that they let her out into normal activity and she recovered.

But the best result is this. Many years later I was told that the great specialist had admitted to friends of his that he had altered his treatment of neurasthenia since reading The Yellow Wallpaper.

It was not intended to drive people crazy, but to save people from being driven crazy, and it worked.

\section{IN OTHER} WORDS
Charlotte Perkins Gilman (18601935) first suffered depression in 1882. After giving birth to a daughter in 1885 her depression worsened. She entered a sanatorium in 1887, under the care of $\mathrm{Dr}$ Silas Weir Mitchell, the American physician, psychiatrist and writer. Gilman recovered but experienced another depressive episode in 1889. She took her own life by chloroform poisoning in 1935, while suffering the final stages of breast cancer. The Yellow Wallpaper was first published in 1892, in The New England Magazine. This excerpt originally from Gilman's own magazine The Forerunner (October 1913) is reprinted in The Yellow Wallpaper (edited by Dale M. Bauer), Bedford Books, 1998, pages 348-349.

doi: 10.1192/apt.17.4.265 